Purdue University

Purdue e-Pubs

CTRC Research Publications

Cooling Technologies Research Center

2005

\title{
A Two-Temperature Model for Solid/Liquid Phase Change in Metal Foams
}

S. Krishnan

J. Y. Murthy

S V. Garimella

Purdue University, sureshg@purdue.edu

Follow this and additional works at: http://docs.lib.purdue.edu/coolingpubs

Krishnan, S.; Murthy, J. Y.; and Garimella, S V., "A Two-Temperature Model for Solid/Liquid Phase Change in Metal Foams" (2005). CTRC Research Publications. Paper 273.

http://dx.doi.org/10.1115/1.2010494

This document has been made available through Purdue e-Pubs, a service of the Purdue University Libraries. Please contact epubs@purdue.edu for additional information. 


\section{A Two-Temperature Model for Solid-Liquid Phase Change in Metal Foams}

\section{Shankar Krishnan}

Jayathi Y. Murthy
Suresh V. Garimella ${ }^{1}$
e-mail: sureshg@ecn.purdue.edu

Cooling Technologies Research Center, School of Mechanical Engineering, Purdue University, West Lafayette, Indiana 47907-2088
Transient solid-liquid phase change occurring in a phase-change material (PCM) embedded in a metal foam is investigated. Natural convection in the melt is considered. Volume-averaged mass and momentum equations are employed, with the BrinkmanForchheimer extension to the Darcy law to model the porous resistance. Owing to the difference in the thermal diffusivities between the metal foam and the PCM, local thermal equilibrium between the two is not assured. Assuming equilibrium melting at the pore scale, separate volume-averaged energy equations are written for the solid metal foam and the PCM and are closed using an interstitial heat transfer coefficient. The enthalpy method is employed to account for phase change. The governing equations are solved implicitly using the finite volume method on a fixed grid. The influence of Rayleigh, Stefan, and interstitial Nusselt numbers on the temporal evolution of the melt front location, wall Nusselt number, temperature differentials between the solid and fluid, and the melting rate is documented and discussed. The merits of incorporating metal foam for improving the effective thermal conductivity of thermal storage systems are discussed. [DOI: 10.1115/1.2010494]

\section{Introduction}

Solid-liquid phase change in porous media occurs in many practical applications, such as thermal energy storage, freezing of moist soils, and manufacture of metal-matrix composites. Of particular interest to this study are thermal energy storage units with thermal conductivity enhancers (e.g., metal foams) for transient thermal control of electronics. Here, the phase-change material (PCM) is impregnated into a metal foam. The latent heat of the PCM serves to absorb the heat generated by the electronics, whereas the metal foam helps transport heat from the heat source into the volume of the PCM and helps utilize the PCM more effectively. It is important to understand the flow, heat transfer, and phase change in these systems to better design phase-change energy storage systems for electronics cooling.

Melting and solidification of pure materials coupled with natural convection in the liquid melt has been studied extensively over the years [1-3]. A detailed review of the literature on solid-liquid phase-change heat transfer is given in [4]. Solid-liquid phasechange processes in porous media have also received wide attention as they have many practical applications. Beckermann and Viskanta [5] performed a combined experimental and numerical investigation of phase change occurring in a porous medium. Experiments were performed in a square enclosure with glass beads saturated with gallium. In their mathematical model, they assumed local thermal equilibrium between the glass beads and gallium. Their numerical results, which showed reasonable agreement with experiments, revealed that the solid-liquid interface shape was profoundly influenced by natural convection in the melt and heat conduction in the solid. Jany and Bejan [6] reported a scaling analysis of melting in porous media with local thermal equilibrium between the porous matrix and the PCM; melt convection was also incorporated. They found that the general behavior of the phase change process with a porous medium was similar to that of phase change without the porous medium. The melting phenomenon in porous media was shown to pass through four distinct

\footnotetext{
${ }^{1}$ Author to whom correspondence should be addressed.

Contributed by the Heat Transfer Division for publication in the Journal of HeAt TRANSFER. Manuscript received July 21, 2004. Final manuscript received November 13, 2004. Review conducted by: N. K. Anand.
}

regimes, each regime being characterized by distinct Nusselt numbers. Bejan [7] reported an analytical study of melting in a confined porous medium saturated with a PCM. Non-Darcian effects were ignored as was the initial subcooling. The local thermal equilibrium assumption was also invoked between the solid and PCM. The liquid Stefan number was found to have a profound effect on the heat transfer and melting rates. Chellaiah and Viskanta studied, numerically and experimentally, the melting of ice in a bed of glass [8] and aluminum [9] beads. They assumed local thermal equilibrium for the numerical model and found reasonable agreement with their experimental results. With the aluminum beads, the agreement between the numerical results and experiments was poor at high Rayleigh numbers, and deviation from local thermal equilibrium was suggested as the cause. A review of the literature on phase-change heat transfer in porous media is available in [10].

Ellinger and Beckermann [11] experimentally investigated the heat transfer enhancement in a rectangular domain partially occupied by a porous layer of aluminum beads. They found that the introduction of a porous layer caused the solid-liquid interface to move faster initially during the conduction-dominated regime. However, the overall melting and heat transfer rates were found to be lower with the porous layer present due to low porosity and permeability. They recommended the use of porous media of higher porosity and permeability with higher Rayleigh numbers for enhancement of melting and heat transfer rates. Tong et al. [12] performed a numerical analysis of the enhancement of melting and heat transfer rates obtained upon incorporating a metal matrix into water, under the assumption of local thermal equilibrium. An order-of-magnitude increase was observed in the heat transfer rate with the metal matrix present. Vesligaj and Amon [13] investigated the passive thermal control of portable electronics using PCMs with thermal conductivity enhancers under unsteady thermal workloads. An epoxy polymer was used as the PCM. The operational performance of portable electronics was found to improve when such a passive thermal storage device was used. Alawadhi and Amon [14] reported numerical and experimental studies on the effectiveness of a thermal control unit composed of an organic PCM and a metal matrix. Modeling the metal matrix using modified effective thermophysical properties was found to yield good agreement with the experiments. Harris et al. [15] presented an approximate theoretical model to analyze the 


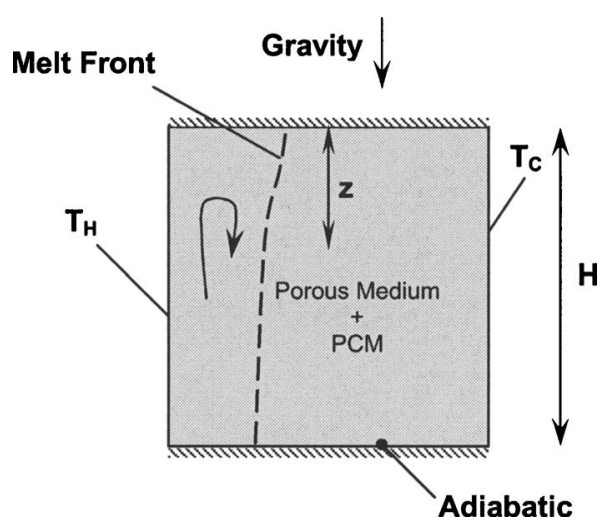

Fig. 1 Schematic of the problem under investigation

phase-change process in a porous medium. Assuming equilibrium melting at the pore scale, a parametric study based on a semiheuristic conduction model was formulated. The conditions for the existence of local thermal equilibrium were explored. A number of studies have discussed the validity of assuming local thermal equilibrium in porous media saturated by a fluid [16-20].

The objective of the present study is to investigate nonequilibrium thermal transport associated with phase change in a rectangular enclosure containing a metal foam impregnated with PCM. Recently, metal foams have been proposed for thermal management of electronics. Applications studied include heat exchanger units [21], thermal storage units [22], and others [23]. Since the thermal conductivity of the phase-change material considered in the present study is two orders of magnitude lower than that of the metal foam, as is typical of organic PCMs, nonequilibrium thermal effects may play a significant role. A two-temperature model is developed, with separate energy equations for the metal foam and the PCM, including phase change in the PCM. This work builds on a previous analysis of natural convection in a PCMfoam domain, but in which change of phase was not considered [16]. Issues investigated and discussed in this work include natural convection in the melt, the effects of Rayleigh, Stefan, and interstitial Nusselt numbers on the temporal evolution of the melt front location, heat transfer rate, the temperature differentials between the solid and fluid, and the melting rate under local thermal nonequilibrium. The results are compared to local thermal equilibrium models, and the validity of the local thermal equilibrium assumption is discussed.

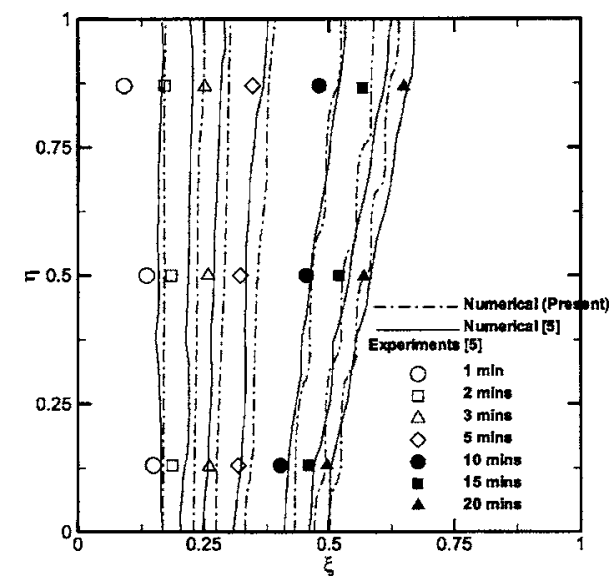

Fig. 2 Comparison of experimentally measured [5] and predicted (from [5] and present work) interface locations at various times
Table 1 Interstitial Nusselt number [26-32]

\begin{tabular}{|c|c|c|c|c|}
\hline & & Interstitial Nusselt Number, $N u_{u, s}=\frac{h_{v} d^{2}}{k_{j}}$ & Analysis Type & Remarks \\
\hline ! & $\begin{array}{l}\text { Wakao and } \\
\text { Kaguei }[26]\end{array}$ & $6(1-\varepsilon)\left[2+1.1(\mathrm{Re})^{0.6}(\mathrm{Pr})^{1 / 3}\right]$ & $\begin{array}{l}\text { Experimental, } \\
\text { Analytical }\end{array}$ & $\begin{array}{c}\text { For forced convertive } \\
\text { flow over spheres } \\
(0<\operatorname{Re}<8500, \varepsilon \sim \\
0.4)\end{array}$ \\
\hline 2 & $\begin{array}{l}\text { Hwang et aj. } \\
\quad[27]\end{array}$ & $0.376(\mathrm{Re})^{0.345}(\mathrm{Pr})^{0.37}$ & Experimental & $\begin{array}{c}\text { For forced flow } \\
\text { across metal foams } \\
(40<\operatorname{Re}<200)\end{array}$ \\
\hline 3 & $\begin{array}{l}\text { Calmidi and } \\
\text { Mahajan [28I }\end{array}$ & $\left(a_{s f} d\right) C \mathrm{Re}^{0.1} \mathrm{Pr}^{0.37}$ & Experimental & $\begin{array}{l}\text { Correlation for forced } \\
\text { flow across metal } \\
\text { foans }(C-0.52 \text {, } \\
40<R e<1000) \text {. } \\
\text { Originalyy proposed } \\
\text { for forced flow across } \\
\text { cylinder. }\end{array}$ \\
\hline 4 & $\begin{array}{c}\text { Phanikumar } \\
\text { and Mahajan } \\
\text { [29] }\end{array}$ & $\left(a_{s f} d\right) \subset \operatorname{Re}^{a s} \mathrm{Pr}^{0,37}$ & Experimenta! & $\begin{array}{c}\text { Conrelation for forced } \\
\text { flow across metal } \\
\text { foams }(\mathrm{C}=0.52 \\
\left.10^{3}<\mathrm{Ra}<5 \times 10^{5}\right) \text {. } \\
\text { Originally proposed } \\
\text { for forced flow across } \\
\text { cylinder. }\end{array}$ \\
\hline 5 & $\begin{array}{l}\text { Kuwwahara et } \\
\text { al. [30] }\end{array}$ & $4(1-\varepsilon)\left[\left(1+\frac{4(1-\varepsilon)}{\varepsilon}\right) \div \frac{1}{2}(1-\varepsilon)^{1 / 2} \operatorname{Re}^{0.5} \mathrm{Pr}^{1 / 3}\right.$ & Numerical & $\begin{array}{c}\text { Forced flow actoss } \\
\text { arbitrary geometry } \\
(0<\operatorname{Re}<8000,0.2< \\
\varepsilon<0.9)\end{array}$ \\
\hline 6 & Morgan [31] & $\frac{2}{\ln \left(\frac{d_{\infty}}{d_{m}}\right)}$ & Analytical & $\begin{array}{l}\text { For an infinitely long } \\
\text { cylinder in an infinite } \\
\text { space. }\end{array}$ \\
\hline 7 & $\begin{array}{l}\text { Frankel and } \\
\text { Acrivos [32] }\end{array}$ & $\frac{2 a_{t f} d}{(1.372+0.5 \ln 2-0.5 \ln (\mathrm{Re}-\mathrm{Pr}))}$ & Analytica! & $\begin{array}{l}\text { For shear flow across } \\
\text { freely suspended } \\
\text { cylinders. The } \\
\text { derived solution is for } \\
\operatorname{Re} . \mathrm{Pr} \rightarrow 0 \text {. }\end{array}$ \\
\hline 8 & $\begin{array}{l}\text { Acrivos and } \\
\text { Taylor [32] }\end{array}$ & $\frac{2}{\ln (\operatorname{Re} \cdot \mathrm{Pr})}$ & Analytical & $\begin{array}{l}\text { For low Reynolds } \\
\text { number flows over } \\
\text { cylinders under } \\
\text { Re.Pr } \rightarrow 0 \text {. }\end{array}$ \\
\hline 9 & $\begin{array}{l}\text { Resistance } \\
\text { Analysis }\end{array}$ & $\frac{a_{y}}{\frac{d}{2 k_{f}}+\frac{d_{m_{-}}}{2 k_{m}}}$ & Analytical & Resistances in series \\
\hline
\end{tabular}

\section{Mathematical Formulation and Numerical Modeling}

A schematic of the problem under investigation is shown in Fig. 1. A square domain of height $H$ encloses the porous metal foam, which is filled with solid phase change material (PCM). The melting point of the PCM is $T_{\text {melt }}^{*}$, and the right wall is maintained at $T_{C}^{*}\left(<T_{\text {melt }}^{*}\right)$. The top and bottom walls are adiabatic. Initially, the solid metal foam and the PCM are at equilibrium at temperature $T_{C}^{*}$. At time $\tau=0$, the left wall temperature is raised to $T_{H}^{*}$ $\left(>T_{\text {melt }}^{*}\right)$ and the PCM is allowed to melt. The thermophysical properties of the solid metal foam and the PCM are assumed to be constant over the range of temperatures considered. In the liquid state, the PCM is assumed to be incompressible, Newtonian, and subject to the Boussinesq approximation. The densities of the solid and liquid PCM are assumed to be equal, i.g., the volume change upon phase change is ignored. Thermal dispersion effects may be important for very high Rayleigh numbers [24], but are neglected in the present work because of the lack of available models for metal foams. The volume-averaged governing mass and momentum equations in dimensionless terms, subject to the above assumptions, are

$$
\begin{aligned}
& \nabla \cdot \mathbf{U}=0 \\
& \frac{1}{\operatorname{Pr}}\left(\frac{1}{\varphi} \frac{\partial \mathbf{U}}{\partial \tau}+\frac{1}{\varphi^{2}}(\mathbf{U} \cdot \nabla) \mathbf{U}\right)=-\nabla \mathbf{P}+\frac{1}{\varphi} \nabla^{2} \mathbf{U}-\left(\frac{1}{\mathrm{Da}^{2}}+\frac{F}{\operatorname{Pr} \cdot \mathrm{Da}}|\mathbf{U}|\right) \mathbf{U} \\
& +\operatorname{Ra} T_{f}^{*} \frac{\mathbf{g}}{|\mathbf{g}|}
\end{aligned}
$$

In Eq. (2), $F$ is the inertial coefficient and $\varphi\left(=V_{l} / V\right)$ is the fraction of liquid PCM in the given volume.

The governing energy equations are written for the metal foam and PCM separately and are closed using an interstitial Nusselt number. Assuming equilibrium melting at the pore scale, i.e., ignoring kinetics effects, the dimensionless energy equations are

$$
(1-\varepsilon) \Omega \frac{\partial T_{m}^{*}}{\partial \tau}=(1-\varepsilon) \lambda \nabla^{2} T_{m}^{*}-\mathrm{Nu}_{i}\left(T_{m}^{*}-T_{f}^{*}\right)
$$




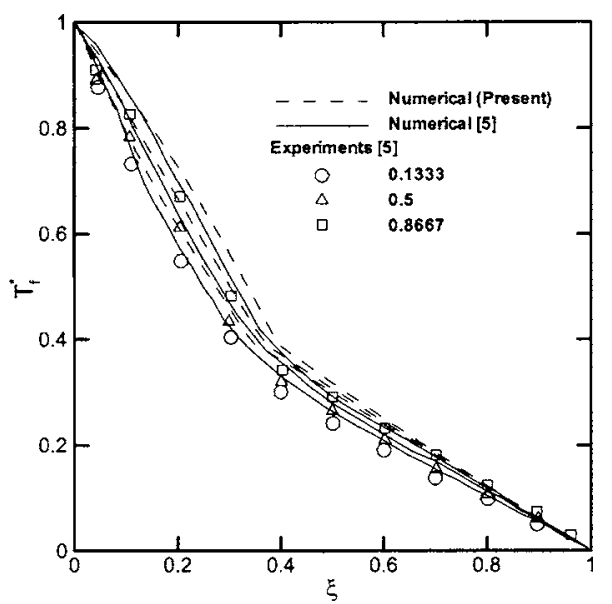

(a)

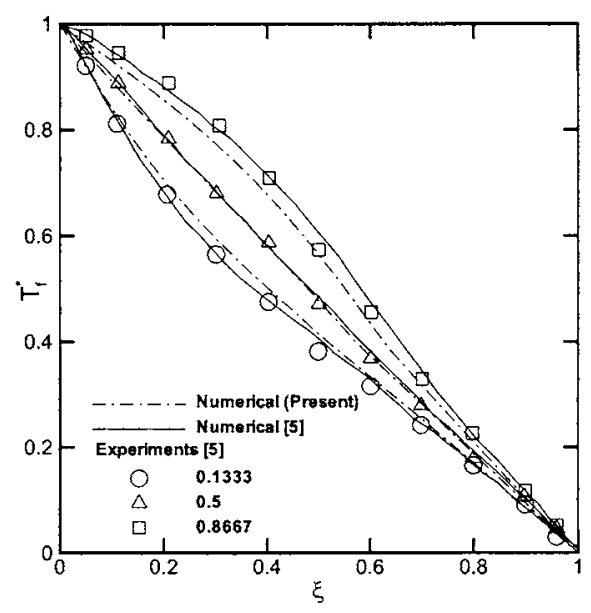

(b)

Fig. 3 Comparison of measured [5] and predicted (from [5] and present work) temperature distributions at three different vertical locations at a dimensionless time of (a) 1.829 (5 min) and $(b) 7.314(20 \mathrm{~min})$

$$
\varepsilon \frac{\partial T_{f}^{*}}{\partial \tau}+(\mathbf{U} \cdot \nabla) T_{f}^{*}=\varepsilon \nabla^{2} T_{f}^{*}-\frac{\varepsilon}{\text { Ste }} \frac{\partial \gamma}{\partial \tau}-\mathrm{Nu}_{i}\left(T_{f}^{*}-T_{m}^{*}\right)
$$

In Eq. (4), $\gamma\left(=V_{l} / V_{f}\right)$ is the fraction of liquid in the PCM. As an alternative, if local thermal equilibrium can be assumed, the energy equations (3) and (4) may be replaced by a single energy equation given by

$$
[\varepsilon+(1-\varepsilon) \Omega] \frac{\partial T^{*}}{\partial \tau}+(\mathbf{U} \cdot \nabla) T^{*}=[\varepsilon+(1-\varepsilon) \lambda] \nabla^{2} T^{*}-\frac{\varepsilon}{\text { Ste }} \frac{\partial \gamma}{\partial \tau}
$$

Equation (5) can be obtained from Eqs. (3) and (4) by setting $T_{m}^{*}=T_{f}^{*}=T^{*}$ and adding Eqs. (3) and (4). If no porous medium were present, the governing mass, momentum, and energy equations would be obtained by setting $\varepsilon=1$ and $\mathrm{Da}=\infty$ in Eqs. (1), (2), and (5). The following dimensionless variables are used in deriving Eqs. (1)-(5):

$$
\xi=\frac{x}{H} ; \quad \eta=\frac{y}{H} ; \quad \tau=\frac{t \alpha_{f}}{H^{2}} ; \quad U=\frac{u H}{\alpha_{f}} ; \quad P=\frac{p H^{2}}{\rho_{f} v_{f} \alpha_{f}}
$$

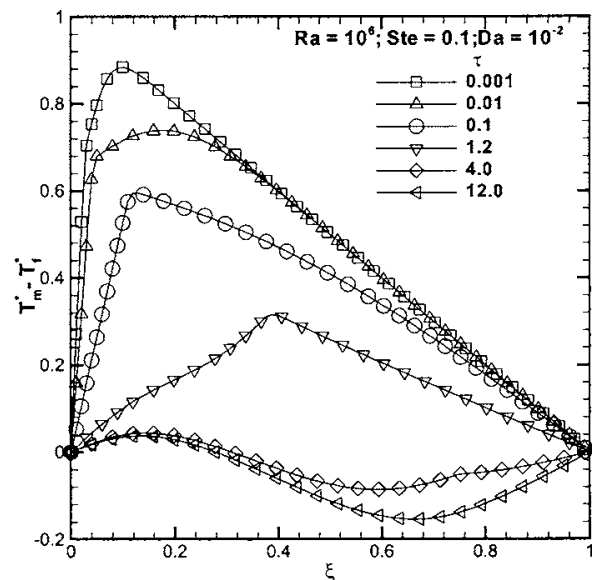

(a)

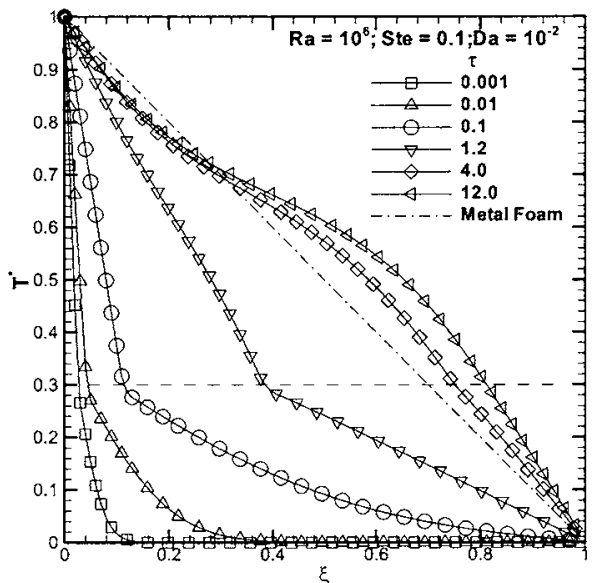

(b)

Fig. 4 Predicted temporal evolution of the thermal field for $\mathrm{Ra}=10^{6}, \mathrm{Nu}_{i}=0, \mathrm{Ste}=0.1, \mathrm{Pr}=50$, and $\mathrm{Da}=10^{-2}$ at the midheight of the domain ( $\boldsymbol{\eta}=0.5$ ): (a) solid-to-fluid temperature difference, and $(b)$ solid and fluid temperature distributions. Also plotted in the figure is the nondimensional melting temperature (horizontal dashed line).

$$
T_{s}^{*}=\frac{\left(T_{m}-T_{C}\right)}{\left(T_{H}-T_{C}\right)} ; \quad T_{f}^{*}=\frac{\left(T_{f}-T_{C}\right)}{\left(T_{H}-T_{C}\right)} ; \quad T^{*}=\frac{\left(T-T_{C}\right)}{\left(T_{H}-T_{C}\right)}
$$

The relationship between $\varphi, \varepsilon$, and $\gamma$ in the above equations can be expressed as follows:

$$
\varphi=\left\{\begin{array}{ll}
\varepsilon & \text { for } T_{f}^{*} \geqslant T_{\text {melt }}^{*}+\Delta T^{*} \\
\varepsilon\left(\frac{T_{f}^{*}-T_{\text {melt }}^{*}+\Delta T^{*}}{2 \Delta T^{*}}\right) & \text { for } T_{\text {melt }}^{*}-\Delta T^{*}<T_{f}^{*}<T_{\text {melt }}^{*}+\Delta T^{*} \\
0 & \text { for } T_{f}^{*} \leqslant T_{\text {melt }}^{*}-\Delta T^{*}
\end{array}\right\}
$$

Here, phase change is assumed to occur over a small but finite temperature range $\Delta T^{*}$ for numerical stability. For all computations performed in this study a $\Delta T^{*}$ of 0.04 or less was used. In order to model solid-liquid phase-change phenomena in the same grid, the third term in Eq. (2) was used to immobilize the material in the fully solid regime by imposing a small value of $K(=1$ $\times 10^{-10} \mathrm{~m}^{2}$ ).

The dimensionless parameters describing the heat and fluid flow processes are

$$
\operatorname{Pr}=\frac{v_{f}}{\alpha_{f}} ; \quad \operatorname{Ra}=\frac{g \beta_{f} H^{3}\left(T_{H}-T_{C}\right)}{\alpha_{f} v_{f}} ; \quad \operatorname{Da}=\frac{\sqrt{K}}{H}
$$




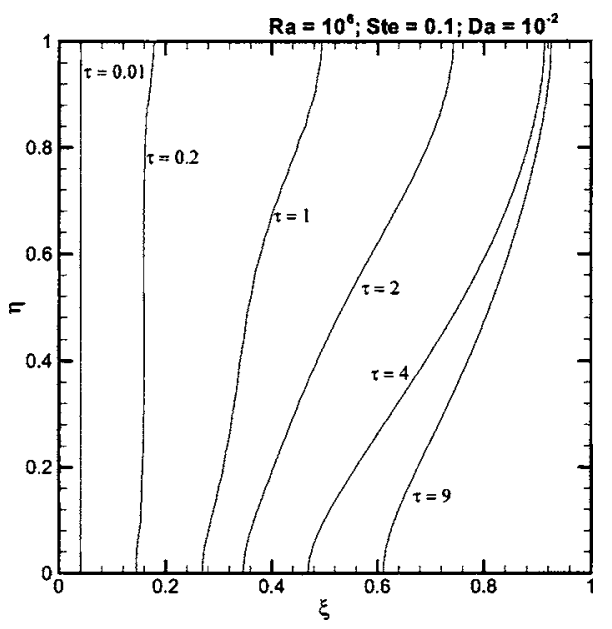

Fig. 5 Predicted temporal evolution of the melt front location for $\mathrm{Ra}=10^{6}, \mathrm{Nu}_{i}=0$, Ste $=0.1, \mathrm{Pr}=50$, and $\mathrm{Da}=10^{-2}$

$$
\begin{gathered}
\mathrm{Nu}_{i}=\frac{h_{v} H^{2}}{k_{f}} ; \quad \text { Ste }=\frac{\left(C_{p}\right)_{f}\left(T_{H}-T_{C}\right)}{\Delta H} \\
\lambda=\frac{k_{m}}{k_{f}} ; \quad \lambda_{e}=\frac{k_{e q}}{k_{f}}=\varepsilon+(1-\varepsilon) \lambda ; \quad \Omega=\frac{\left(\rho C_{p}\right)_{m}}{\left(\rho C_{p}\right)_{f}} ; \quad F ; \quad \varepsilon
\end{gathered}
$$

The effective conductivity is given by $k_{e q}=\varepsilon k_{f}+(1-\varepsilon) k_{m}$. The inertial coefficients $F$ for flow over a packed bed of particles [16] and metal foams [25], are $1.75 / \sqrt{150 \varepsilon^{3}}$ and 0.068 , respectively. For metal foams, a constant porosity of 0.8 is used for all the computations.

The computational domain is discretized into finite volumes using a rectangular mesh. All the variables are stored at the cell centroids. A central-differencing scheme with a deferred correction [26] is used for convective fluxes. A central-differencing scheme is used for discretizing diffusive fluxes as well. The transient terms are discretized using a second-order-accurate threetime-level scheme [26]. The SIMPLE algorithm is used for obtaining the velocity fields, and the linearized systems of equations are solved using a strongly implicit procedure (SIP). The calculations are terminated when the dimensionless residual [26] has dropped at least below $10^{-4}$ for all governing equations.

\section{Code Validation}

The code used in this work was previously validated [16] against the available numerical and experimental studies on natural convection in porous enclosures, but without phase change. Since experimental results are not available in the literature for solid-liquid phase change in metal foams, the code was validated against a study of melting of gallium in a packed bed of glass spheres [5]. The height of the square domain was $4.76 \mathrm{~cm}$ and the porosity of the enclosure was 0.385 . The boundary conditions were the same as illustrated in Fig. 1. Further details about the experiments and the numerical model are available in [5]. The dimensionless governing parameters used for the present computations for this comparison are $\mathrm{Ra}=8 \times 10^{5}, \mathrm{Da}=3.7 \times 10^{-3}$, Ste $=0.124, \operatorname{Pr}=0.0208, d / H=0.126, \lambda=0.046, \Omega=0.786$, and $T_{m}^{*}$ $=0.3912$. The permeability for liquid flow through a packed bed of spherical particles is calculated from [5]

$$
K(\varphi)=\frac{d^{2} \varphi^{3}}{175(1-\varphi)^{2}}
$$

For closing the two energy equations (3) and (4), the correlation proposed by Wakao and Kaguei [27] was used and is given below

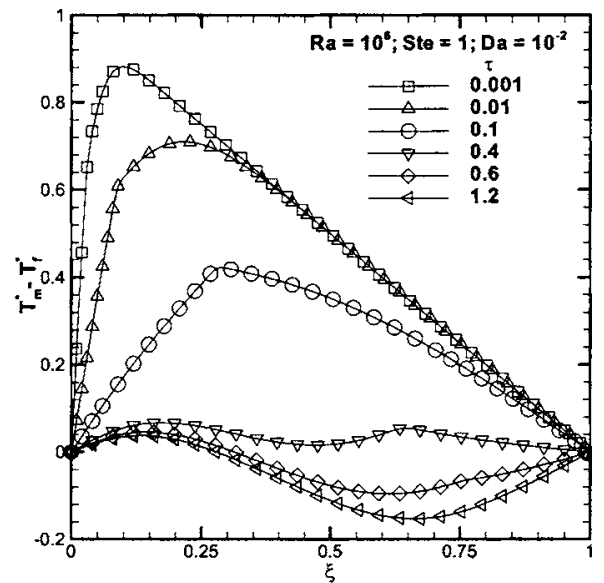

(a)

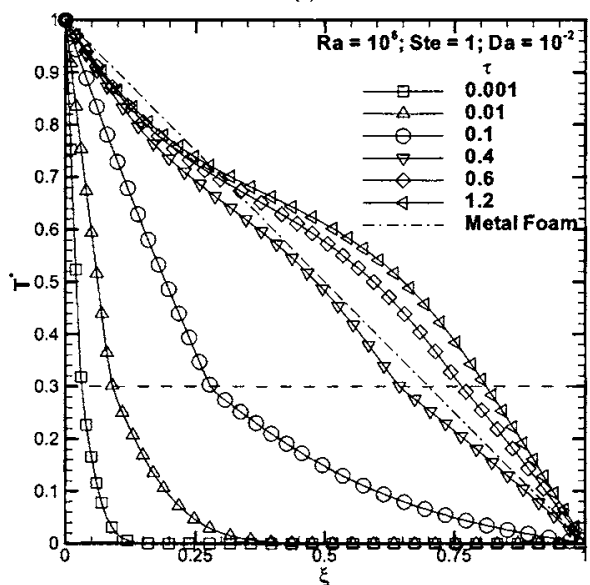

(b)

Fig. 6 Predicted temporal evolution of thermal field for $\mathbf{R a}$ $=10^{6}, \mathrm{Nu}_{i}=0, \mathrm{Ste}=1.0, \mathrm{Pr}=50$, and $\mathrm{Da}=10^{-2}$ at the midheight of the domain $(\eta=0.5)$ : (a) Solid-to-fluid temperature difference, and (b) Solid and fluid temperature distributions. Also plotted in the figure is the nondimensional melting temperature (horizontal dashed line).

$$
\mathrm{Nu}_{i, d}=6(1-\varepsilon)\left[2+1.1(\operatorname{Re})^{0.6}(\operatorname{Pr})^{1 / 3}\right]
$$

This correlation covers a broad range of Reynolds numbers and asymptotes to a constant value for the diffusion-only limit ( Re $\rightarrow 0$ ) for spheres. Though this correlation was reported for forced convection, it is assumed to be approximately valid for natural convection in the melt as well. The Reynolds number in Eq. (6) is interpreted as the local Reynolds number based on the local mean velocity, i.e., $u_{\text {mean }} d / v$. Figure 2 shows the comparison of the computed melt front locations from the present study with those in [5]. The primary difference in the present mathematical model from that in [5] is the relaxation of the assumption of local thermal equilibrium between the solid and the interstitial fluid. The temperature distributions predicted from the present study are compared to those in [5] in Fig. 3 at different vertical ( $\eta$-direction) locations at two different times. The current predictions show satisfactory agreement with experiments and also agree reasonably well with the numerical predictions in [5], despite the assumption of thermal equilibrium in the latter. This agreement between the numerical results can be attributed to the fact that the system is conduction dominated $\left(\mathrm{RaDa}^{2} \sim 11\right)$.

Grid independence of the solution for the meshes used in the present simulations was also established. A Rayleigh number of $10^{6}$, Darcy number of $10^{-2}$, Prandtl number of 50, Stefan number of 0.1 , and porosity of 0.8 were used for this set of calculations. 


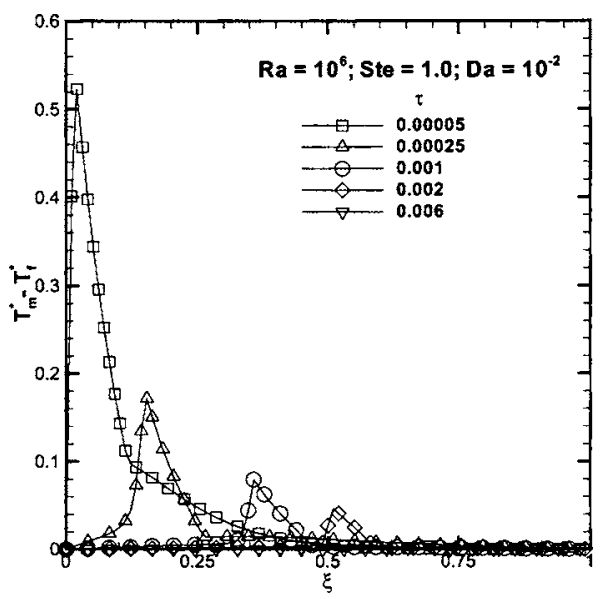

(a)

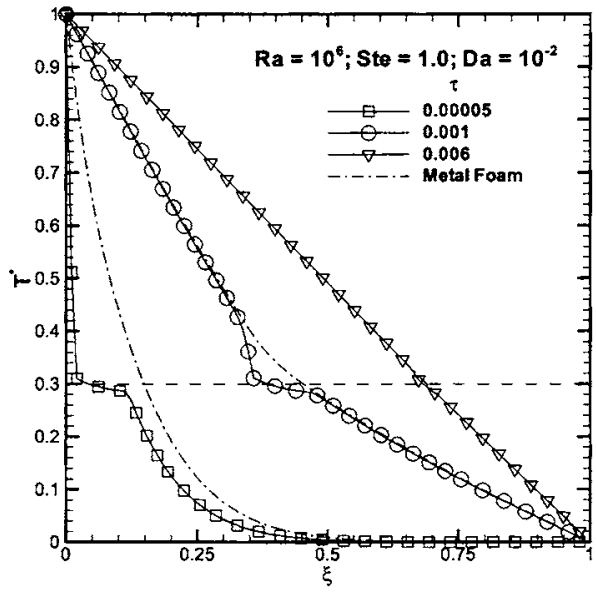

(b)

Fig. 7 Predicted temporal evolution of thermal field for Ra $=10^{6}, \mathrm{Nu}_{i d}=5.9$, Ste $=1.0, \mathrm{Pr}=50$, and $\mathrm{Da}=10^{-2}$ at the midheight of the domain $(\eta=0.5)$ : (a) Solid-to-fluid temperature difference, and (b) solid and fluid temperature distributions. Also plotted in the figure is the nondimensional melting temperature (horizontal dashed line).

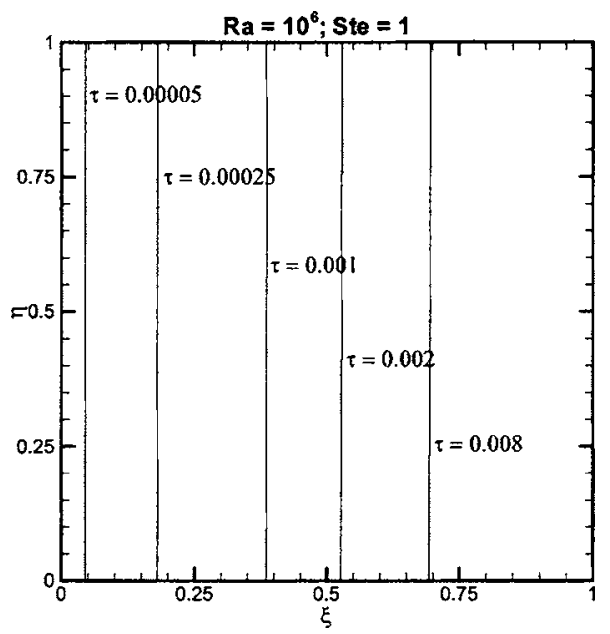

Fig. 8 Predicted temporal evolution of melt front locations $(\gamma$ $=0.5$ ) for $\mathrm{Ra}=10^{6}, \mathrm{Nu}_{i, d}=5.9$, Ste $=1.0, \mathrm{Pr}=50$ and $\mathrm{Da}=10^{-2}$

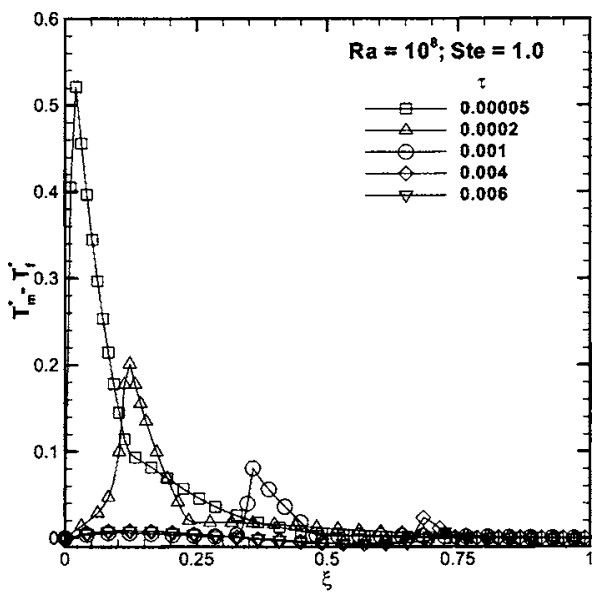

(a)

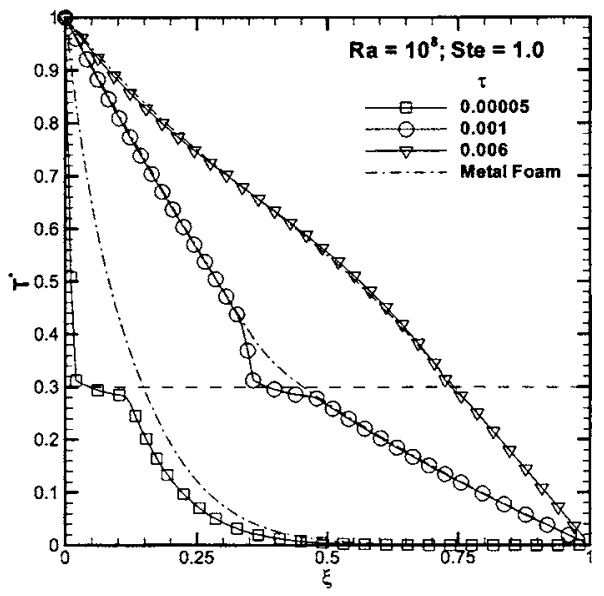

(b)

Fig. 9 Predicted temporal evolution of thermal field for $\mathbf{R a}$ $=10^{8}, \mathrm{Nu}_{i, d}=5.9, \mathrm{Ste}=1.0, \mathrm{Pr}=50$, and $\mathrm{Da}=10^{-2}$ at the midheight of the domain ( $\eta=0.5)$ : (a) Solid-to-fluid temperature difference, and (b) solid and fluid temperature distributions. Also plotted in the figure is the nondimensional melting temperature (horizontal dashed line).

Grid sensitivity was tested on three different grids, $48 \times 48$ (grid 1), $102 \times 102$ (grid 2), and $186 \times 186$ (grid 3). For grid 1, deviations of $7.5 \%$ and $2 \%$ were observed, respectively, in the minimum and maximum solid-to-fluid temperature differences with respect to grid 3 at time $\tau=0.225$. For grid 2 at $\tau=0.225$, the corresponding deviations with respect to grid 3 reduced to $1 \%$ and $1.7 \%$. The melt front shapes were almost identical in these latter two cases. The intermediate grid of $102 \times 102$ was found to be sufficient for all the calculations presented in this study.

\section{Results and Discussion}

The porosity of the metal foam considered is held constant at 0.8 and the pore size $(d / H)$ is 0.0135 . The typical ratio of the average ligament diameter $d_{m}$ of the foam to the mean cell size $d$ is 0.1875 , with the average ligament diameter of $0.36 \mathrm{~mm}$ [25]. The ratio of the metal foam-to-PCM thermal conductivity $\lambda$ is 1000. The Prandtl number of the PCM is fixed at 50, which is typical of the value for many organic PCMs.

A correlation similar to Eq. (6) (which was for spherical beads) that covers a broad range of Reynolds numbers was not found in the literature for metal foams. For high-Prandtl-number fluids undergoing phase change in porous enclosures the velocities encountered are small (e.g., the vertical velocity $v(\mathrm{~m} / \mathrm{s})$ is $O\left(10^{-3}\right)$ or 


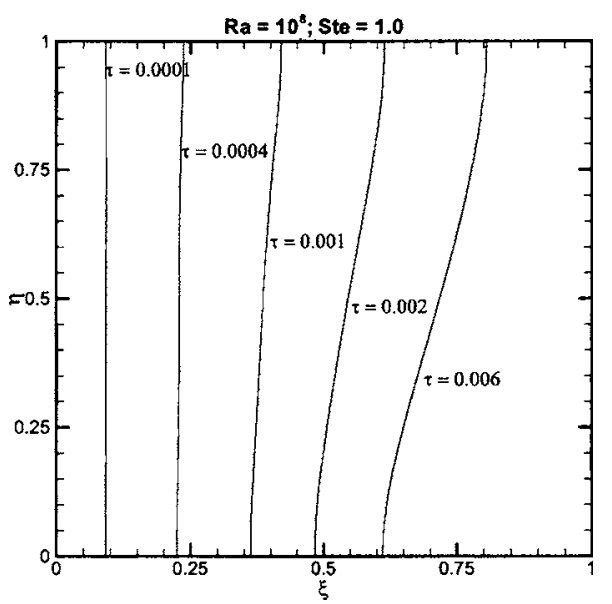

Fig. 10 Predicted temporal evolution of melt front locations $(\gamma=0.5)$ for $\mathrm{Ra}=10^{8}, \mathrm{Nu}_{i, d}=5.9, \mathrm{Ste}=1.0, \operatorname{Pr}=50$, and $\mathrm{Da}=10^{-2}$

less for $\mathrm{RaDa}^{2} \leqslant 10^{4}$ ). Also, the heat transfer between the metal foam and the solid PCM is only by conduction during the initial transient. As the system is largely conduction dominated, it is critical to establish the diffusion limit for the interstitial heat transfer coefficients. Table 1 [25,27-32] lists the various models used in the present study for closing the energy equations (3) and (4). Though some of the correlations in Table 1 are for forced convection, they are assumed to be approximately valid for natural convection in the melt as well. The Reynolds number in the equations is interpreted as the local Reynolds number based on the local mean velocity, i.e., $u_{\text {mean }} d / v$. As $\operatorname{Re} \rightarrow 0$, some of the equations yield $\mathrm{Nu}_{i, d}=0$, which does not correctly represent the conduction limit. In the equations listed in Table $1, a_{s f}$ is the specific surface area of the metal foams and is given by the relation [28]

$$
a_{s f}=\frac{3 \pi d_{m}}{0.59 d^{2}}\left\{1-\exp \left[-\left(\frac{1-\varepsilon}{0.04}\right)\right]\right\}
$$

In the discussion that follows, the "fluid" phase refers to the PCM, whether solid or melted, whereas the "solid" phase refers to the metal foam. In order to understand the effect of interphase heat transfer on the melting of PCM, the case of zero interphase heat transfer $\left(\mathrm{Nu}_{i}=0\right)$ is first considered. Under this condition, there is no thermal interaction between the metal foam and the PCM, and the melt experiences only the flow resistance offered by the porous foam. Figure 4(a) shows the temporal evolution of the dimensionless solid-to-fluid temperature difference along $\eta=0.5$ for $\mathrm{Ra}=10^{6}, \operatorname{Pr}=50, \mathrm{Da}=10^{-2}, \lambda=10^{3}, \Omega=1$, and $T_{\text {melt }}^{\prime}=0.3$. The Stefan number for this case is 0.1 . Figure $4(b)$ shows the PCM and metal foam temperature distributions at the midheight of the domain. In Fig. 4(b) and in other similar figures that follow, the dimensionless metal foam temperature distribution is represented using dotted-dashed lines, whereas the PCM is represented using solid lines with open symbols. Also, the dimensionless melting point is shown as a horizontal dashed line. In the limit of zero interphase heat exchange, the metal foam and PCM develop independently and the temperature difference between them is determined by the relative response of the two phases. The metal foam reaches a steady state in a time scale of order $\left(H^{2} / \alpha_{m}\right)$, or a dimensionless time scale of $\tau_{m} \sim \Omega / \lambda$. For low Rayleigh numbers and Ste $<1$, the time for the PCM to reach a steady state is governed by the effective inertia associated with phase change. It scales as $H^{2} / \alpha_{e f}$ where $\alpha_{e f}=k_{f} /\left(\rho_{f} C_{p_{e f}}\right)$ and $\left(C_{p}\right)_{e f}=(\Delta H \varepsilon) / \Delta T$. The corresponding dimensionless time scale is $\tau_{f} \sim \varepsilon /$ Ste. The metal foam is seen to have reached a steady state well before the temperature field in the fluid has started to develop. Thus at early times, the temperature differential in the domain is very large, of $O(1)$. The temperature difference progressively decreases with

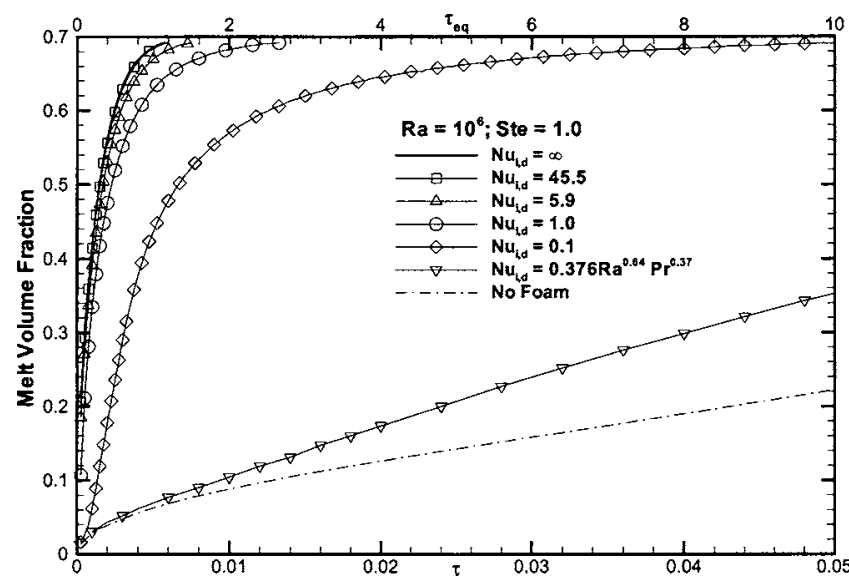

Fig. 11 Predicted melt volume fractions as a function of $\tau$ for $\mathrm{Ra}=10^{6}, \mathrm{Da}=10^{-2}$, Ste $=1.0$, and $\mathrm{Pr}=50$ for various interstitial Nusselt numbers $\left(\mathrm{Nu}_{i, d}\right)$. Also shown are the $\tau_{e q}\left(=\alpha_{e q} t / H^{2}\right)$ values for comparison.

time, reaching a steady-state value over the PCM time scale. Furthermore, the maximum temperature difference between the metal foam and PCM occurs near the solid-liquid interface. This is expected because the phase-change process constrains the PCM temperature to $T_{\text {melt }}^{*}$ at the interface. The thickness of the warm boundary layer $\left(\sim\left[\mathrm{RaDa}^{2}\right]^{-0.25}\right)$ near the heated wall ceases to grow at a time of order $\varepsilon /\left(\operatorname{Ste} \sqrt{\mathrm{RaDa}^{2}}\right)$ [3]. This is the time at which a convective regime in the melt [3] sets in. The time predicted for the onset of the convective regime by Benard et al. [33] is $4.59 \varepsilon /\left(\mathrm{Ste} \sqrt{\mathrm{RaDa}^{2}}\right)$.

Figure 5 shows the temporal evolution of melt front location for $\mathrm{Nu}_{i}=0, \mathrm{Ra}=10^{6}, \mathrm{Ste}=0.1, \mathrm{Da}=10^{-2}, \mathrm{Pr}=50, \lambda=10^{3}, \Omega=1$, and $T_{\text {melt }}^{*}=0.3$. Initially, the process is conduction-dominated and the melt front is planar. The horizontal intrusion layer appears in the top half of the domain at a dimensionless time of 0.2 because of the development of natural convection. This denotes the beginning of the convection-plus-conduction regime [3]. The vertical height (see Fig. 1) of the upper portion of the domain $z$, where convection dominates conduction scales as $\left(\operatorname{RaDa}^{2}\left[\tau_{f} \mathrm{Ste} / \varepsilon\right]^{2}\right)[3]$. At a nondimensional time of approximately $\varepsilon /\left(\operatorname{Ste} \sqrt{\mathrm{RaDa}^{2}}\right)$, the thermal boundary layer ceases to grow and the quasi-steady regime commences. This can also be seen from Figs. $4(a)$ and $4(b)$, where the boundary layer thickness is approximately the same after a nondimensional time of 1.2. In this quasi-steady regime, the movement of the melt front is slow enough that the convective process reaches a steady state before the melt front moves to any significant extent.

The predicted dimensionless solid-to-fluid temperature difference for a higher Stefan number of 1.0 at different times along $\eta=0.5$ for all other parameters remaining the same as above $\left(\mathrm{Nu}_{i}=0, \mathrm{Ra}=10^{6}, \mathrm{Da}=10^{-2}, \mathrm{Pr}=50, \lambda=10^{3}, \Omega=1\right.$, and $\left.T_{\text {melt }}^{*}=0.3\right)$ are shown in Fig. 6(a). As expected, the increase in Stefan number from 0.1 in Fig. 4 to 1.0 in Fig. 6 expedites the melt front propagation. As noted previously, the metal foam reaches a steady state over a dimensionless time of $\Omega / \lambda$ and the PCM reaches a steady state over a dimensionless time of $\varepsilon /$ Ste. A comparison of Figs. $4(b)$ and $6(b)$, in which the temporal evolution of the solid and fluid temperature distributions along $\eta=0.5$ is plotted, reveals that the temperature distributions at steady state are identical for the two different Stefan numbers, as expected from Eq. (4). The melt front location for $\mathrm{Ste}=1.0$ is similar to that in Fig. 5, except for the faster evolution of the melt interface, and hence is not shown for brevity. For cases of practical interest, the Stefan number is not large enough to compete with the time scale for the evolution of 


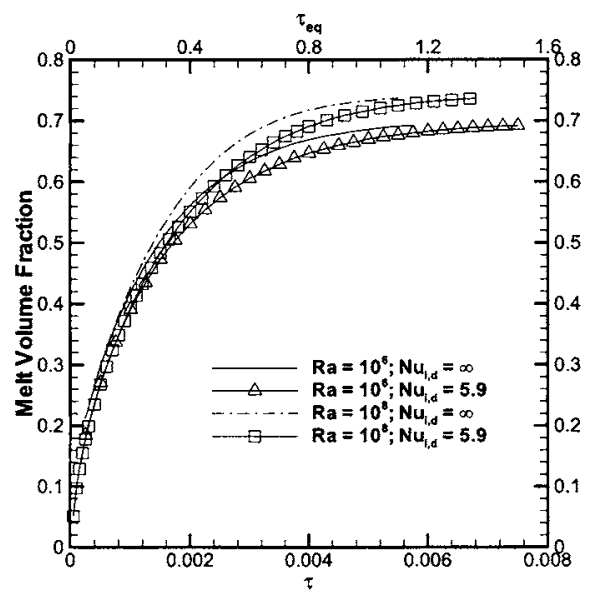

(a)

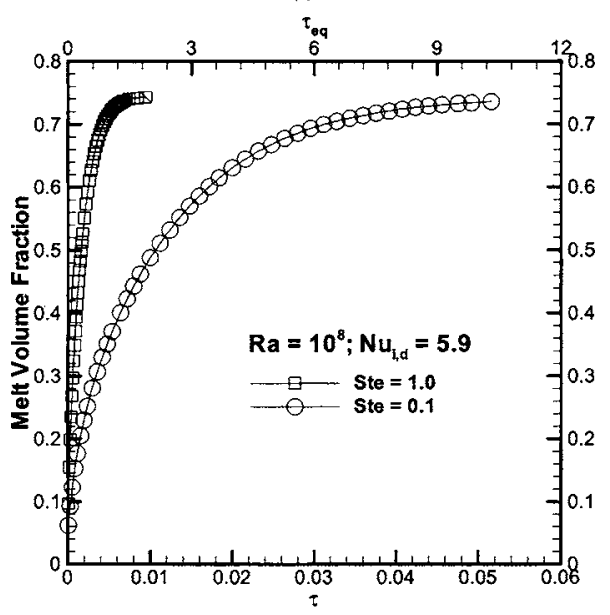

(b)

Fig. 12 Predicted melt volume fractions as a function of $\tau$ for different Rayleigh, Stefan, and interstitial Nusselt numbers. Also shown are the $\tau_{e q}$ values for comparison.

the solid temperature profile, and the overall behavior described in Figs. 5 and 6 is expected to be typical for $\mathrm{Nu}_{i}=0$.

The next case considered is one for which the metal foam and $\mathrm{PCM}$ exchange energy through interphase transfer $\left(\mathrm{Nu}_{i} \neq 0\right)$. Figure 7(a) shows the dimensionless solid-to-fluid temperature difference at various times along $\eta=0.5$, for $\mathrm{Ra}=10^{6}$, Ste $=1.0$, Da $=10^{-2}, d / H=0.0135, \operatorname{Pr}=50, \lambda=10^{3}, \Omega=1$, and $T_{m}^{*}=0.3$. The interstitial Nusselt number $\left(\mathrm{Nu}_{i, d}\right)$ based on the pore diameter used for the calculations is 5.9. This Nusselt number was obtained from the expression by Morgan [31] in Table 1 using a $d_{\infty} / d_{m}$ ratio of $10^{4}$. Figure $7(b)$ shows the dimensionless metal foam and PCM temperature distributions along $\eta=0.5$ for the same set of parameters. The metal foam-to-PCM temperature difference is determined by the heat exchange between the metal foam and PCM. If the response time for the heat exchange between the PCM and metal foam $\left(\tau_{i} \sim\left[(1-\varepsilon) \Omega / \mathrm{Nu}_{i}\right]\right)$ is long compared to the response times of the two phases, the two phases evolve separately, large solid-to-fluid temperature differences exist, and the time to steady state is determined by the slower phase. On the other hand, if the response time for heat exchange is short compared to the response times of the two phases, then the two phases develop together in a coupled manner. For $\mathrm{Nu}_{i}>[(1-\varepsilon) \lambda]$, the interphase exchange time is shorter than the diffusion time for metal foam. For the case in Fig. 7, the interphase exchange time is shorter than both the diffusion time for metal foam as well as that for the PCM (i.e., $\left.\tau_{i}<\tau_{m}, \tau_{f}\right)$ and hence the metal foam and the PCM develop together in a coupled manner. Initially, the metal foam-to-PCM tem-

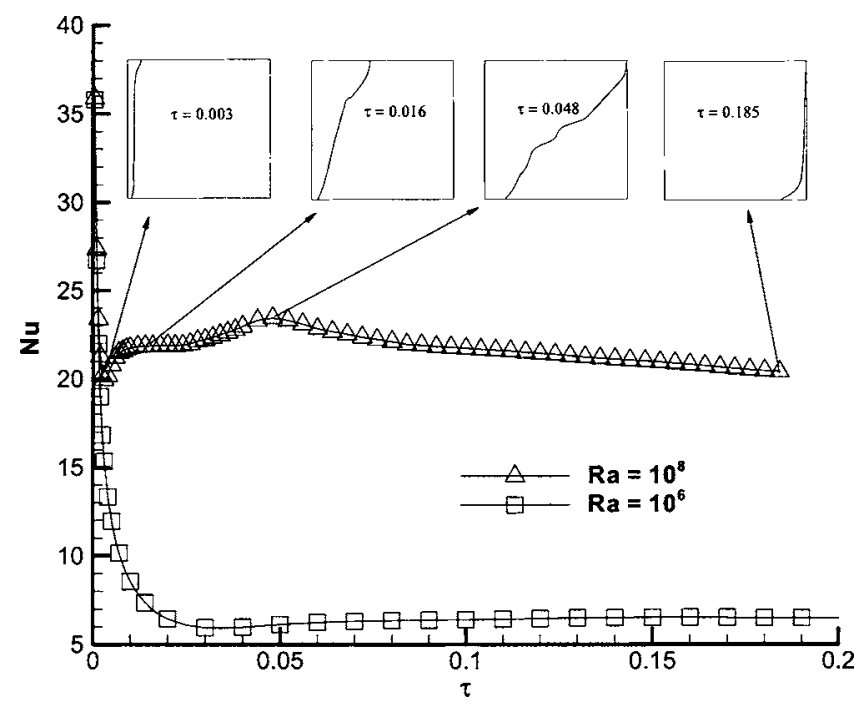

Fig. 13 Predicted hot wall Nusselt number for the no-foam case as a function of $\tau$ for $\mathrm{Ste}=1$ and two different Rayleigh numbers. Also shown are the melt fronts at several critical time instants during flow evolution.

perature difference is very high, but it progressively decreases with time and is driven to a value of zero at steady state. It may be noted that a steady state is reached on a time scale of $O(\Omega / \lambda)$. In the earlier case of $\mathrm{Nu}_{i}=0$, the fluid phase was seen to respond on a time scale of $\tau \sim O(1)$. Here, however, the fluid is seen to evolve faster, on a scale closer to that of the metal foam, as a result of interface exchange. Initially, the metal foam responds faster than the PCM at the hot wall boundary. But since the heat exchange time is shorter than the diffusion scale $\tau_{f}$ for the PCM, heat from the hot boundary travels through the metal and the metal temperature imprints itself on the fluid. Also, as seen in the previous case of $\mathrm{Nu}_{i}=0$, the maximum solid-to-fluid temperature difference occurs at the interface, which is held at $T_{m}^{*}$ because of phase change. It may also be noted that the dimensionless solid-to-fluid temperature difference for the uncoupled (Fig. 4) case is not zero at steady state, whereas in the present case, a zero temperature difference is obtained at steady state. Since the fluid temperature distribution is linear across the domain, the amount of melted PCM is less for $\mathrm{Nu}_{i} \neq 0$ than that of $\mathrm{Nu}_{i}=0$; this aspect will be discussed, in detail, later in this paper, as will the effect of variations in the interstitial Nusselt number and Stefan number.

The predicted temporal evolution of the front location for $\mathrm{Ra}$ $=10^{6}, \mathrm{Nu}_{i, d}=5.9, \mathrm{Ste}=1.0, \mathrm{Pr}=50$, and $\mathrm{Da}=10^{-2}$ is shown in Fig. 8. Contours of the liquid fraction, $\lambda=0.5$ corresponding to the melting temperature $\left(T_{\text {mell }}^{*}\right)$ of 0.3 , are plotted in Fig. 8 . It may be noted, initially at $\tau=0.00005$, from Fig. $7(b)$ that the mushy zone "thickness" is large, i.e., the mushy zone occupies several computational cells because of the presence of the metal matrix. Eventually at steady state $(\tau=0.006)$, the mushy zone thickness is confined to a single computational cell. It can be seen from Fig. 8 that the heat transfer process is conduction dominated. The melt front shape is planar throughout the melting process and little natural convection is observed when compared to the $\mathrm{Nu}_{i}=0$ case in Fig. 5.

The effect of increasing Rayleigh number on the dimensionless solid-to-fluid temperature difference is discussed next. Figure $9(a)$ shows the dimensionless solid-to-fluid temperature difference along $\eta=0.5$ for $\mathrm{Ra}=10^{8}, \mathrm{Da}=10^{-2}, \mathrm{Ste}=1, \mathrm{Pr}=50$, and $\mathrm{Nu}_{i, d}$ $=5.9$. Figure $9(b)$ shows the dimensionless temperature distributions in the metal foam and PCM along $\eta=0.5$ for these parameters. The overall behavior is similar to the $\mathrm{Ra}=10^{6}$ case, albeit with greater convective effects. The solid and fluid temperature 


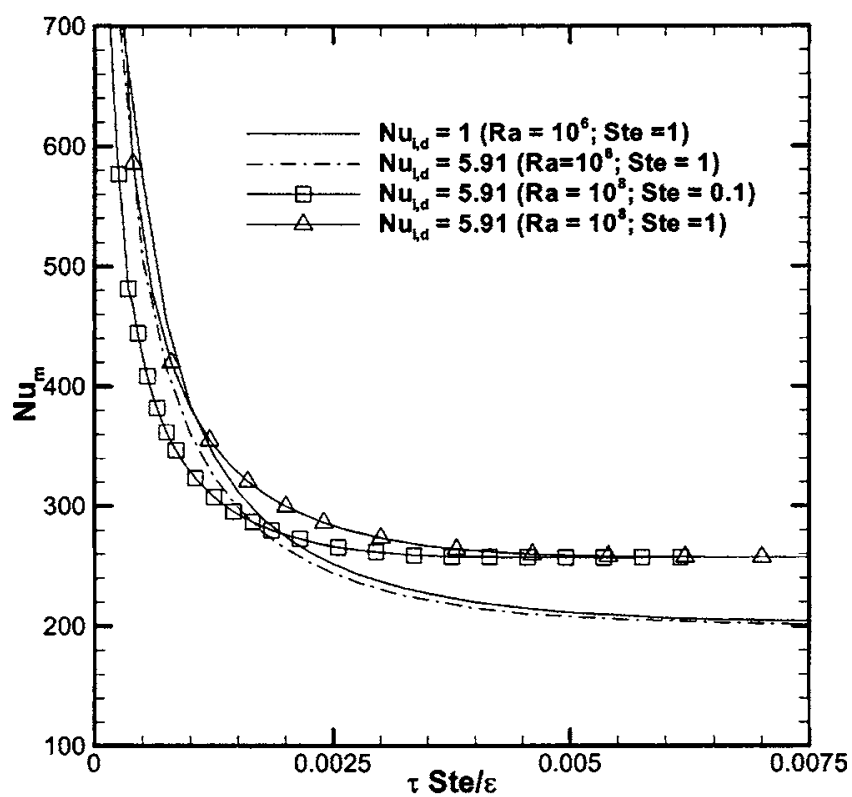

(a)

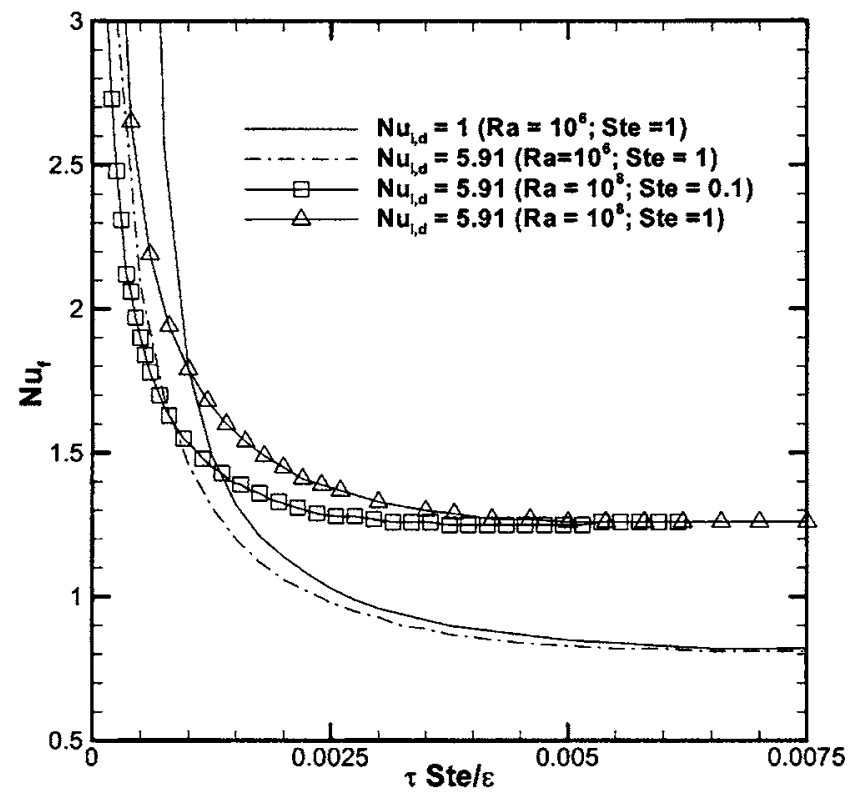

(b)

Fig. 14 Predicted hot wall Nusselt number for (a) metal foam and (b) PCM for various Rayleigh, Stefan, and interstitial Nusselt numbers

profiles are seen to be nearly identical at steady state and exhibit curvature due to convection. The system is driven to steady state on a time scale $O(\Omega / \lambda)$. Figure 10 shows the temporal evolution of the melt front locations for $\mathrm{Ra}=10^{8}, \mathrm{Da}=10^{-2}$, Ste $=1, \mathrm{Pr}=50$, and $\mathrm{Nu}_{i, d}=5.9$. The influence of natural convection on the melt front shape is clear.

A detailed discussion of temperature profiles and melt front shapes for a different $\mathrm{Nu}_{i}$ correlation is outlined in [34]. It is not included here for brevity.

Melt Volume Fraction. From an engineering standpoint, the melt volume fraction and time-dependent average melt front location are of interest in foam-enhanced PCM units. The effect of interstitial heat transfer coefficient on the melted volume is first discussed. Figure 11(a) shows the melt volume fraction $\left(=\Sigma_{\text {cells }} \gamma \Delta x \Delta y\right)$ as a function of $\tau$ for $\mathrm{Ra}=10^{6}, \mathrm{Ste}=1, \mathrm{Da}=10^{-2}$,
$\operatorname{Pr}=50$, and for different interstitial Nusselt numbers (obtained from various $\mathrm{Nu}_{i}$ relations listed in Table 1 for $\varepsilon=0.8$ ). Also plotted in the figure for comparison is the nondimensional time $\tau_{e q}$ based on the effective conductivity of the system (foam + PCM). As expected, the higher the interstitial Nusselt number, the faster is the rate at which a steady state is achieved. The time to steady state is dictated by the metal foam response time, as discussed previously. For the parameters considered here, the final steadystate profile for both solid and fluid is a straight line for all $\mathrm{Nu}_{i, d}$, and the melt volume fraction is thus the region $T^{*}>T_{\text {melt }}^{*}$ Consequently, the melt volume fraction is the same for all the different $\mathrm{Nu}_{i, d}$ values, with the asymptote being $\left(1-T_{\text {melt }}^{*}\right)$. For values of $\mathrm{Nu}_{i, d} \geqslant 1$, little difference in the time to steady state is seen as $\mathrm{Nu}_{i, d}$ is increased. For $\mathrm{Nu}_{i, d}>5.9$, the transient response is not particularly distinguishable from that of the equilibrium model $\left(\mathrm{Nu}_{i, d} \rightarrow \infty\right)$.

Though not shown here, the melt volume fraction at steady state for $\mathrm{Nu}_{i}=0$ is 0.8 for the parameter set in this study. For the case when there is no foam, the melt volume fraction is 0.92 at steady state. In the presence of the foam, convective flow in the melt is retarded due to low values of the parameter $\mathrm{RaDa}^{2}$ $(=100)$ and conduction-dominated profiles result, limiting the melt volume fraction to $\left(1-T_{\text {melt }}^{*}\right)$. But for the uncoupled $\left(\mathrm{Nu}_{i}=0\right)$ and the no-foam cases, the fluid temperature distribution is not linear at steady state and convection in the fluid increases the overall melted volume. However, the response time of the system is substantially slower than for the $\mathrm{Nu}_{i, d}>0$ cases. In practical terms, this means less effective cooling during the transient.

In Fig. 12(a) the effect of varying the Rayleigh number on the evolution of the melt volume fraction is shown. Also plotted in Fig. 12 is $\tau_{e q}$ as in Fig. 11. As the Rayleigh number increases, the convection contribution increases and the melting rate and the melt volume fraction of the PCM also increase somewhat. In Fig. $12(b)$, the effect of decreasing the Stefan number is examined. A decrease in Stefan number from 1.0 to 0.1 with $\mathrm{Ra}=10^{8}$ decreases the rate of melting due to the thermal inertia associated with phase change and the concomitant increase in the latent heat of fusion. Both cases are computed upto a melt volume fraction of 0.74

Wall Nusselt Number. In order to compare the performance of the PCM with and without the metal foam, the average Nusselt number at the hot wall for the case without the metal foam case is discussed. The average Nusselt number at the wall is defined as

$$
\mathrm{Nu}=\frac{h H}{k_{f}}=(-1) \int_{0}^{1}\left[\frac{\partial T^{*}}{\partial \xi}\right]_{\xi=0} d \eta
$$

In the above equation, $T^{*}$ is the nondimensional PCM temperature. Figure 13 shows the average Nusselt number at the hot wall for two different Rayleigh numbers $\left(10^{6}\right.$ and $\left.10^{8}\right)$ and $S t e=1.0$ as a function of dimensionless time. Also plotted in Fig. 13 are the melt front locations for $\mathrm{Ra}=10^{8}$ for different flow regimes. Initially, at $\tau=0$, when the left wall temperature is raised to $T_{H}$, the heat transfer from the wall to the PCM is large, but the Nusselt number falls rapidly as the PCM heats up. Over a time scale $\tau$ $\sim\left(1-T_{\text {melt }}^{*}\right)^{5 / 4} \mathrm{Ra}^{-1 / 2}[3]$, the Nusselt number reaches a low value corresponding to the pure conduction limit $[3,34]$ and starts to increase as convection sets in (referred to as conduction-plusconvection regime in [3]). The increase in $\mathrm{Nu}\left(\sim \tau^{-0.5}+\mathrm{Ra}(\tau)^{3 / 2}\right.$ [3]) in the convection-plus-conduction regime is very small for $\mathrm{Ra}=10^{6}$. The "quasi-steady" regime or the convection-dominated regime sets in over a time scale $\tau \sim(\mathrm{Ra})^{-1 / 2}$ [3]. The Nusselt number is constant in this regime and is computed to be approximately 22 and 7 for $\mathrm{Ra}=10^{8}$ and $10^{6}$, respectively. The Nusselt number starts to increase again as the melt front nears the cold wall and peaks when the melt front touches the cold wall on a time scale $\tau$ of $O\left(\mathrm{Ra}^{-0.25}\right)$ [3]. The Nusselt number drops again 


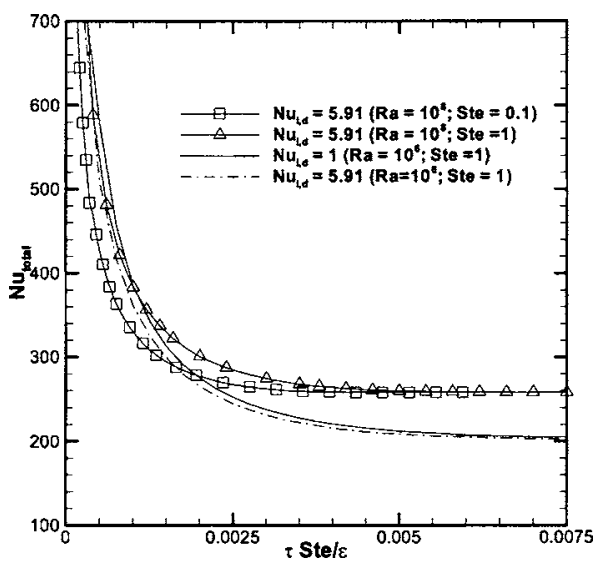

Fig. 15 Predicted total hot wall Nusselt number for various Rayleigh, Stefan, and interstitial Nusselt numbers

when the melt front traverses along the right wall.

The behavior in the presence of the metal foam is markedly different. Figure 14 shows the Nusselt number at the hot wall in this case. The Nusselt number corresponding to the metal foam, $\mathrm{Nu}_{m}$, and that corresponding to the PCM, $\mathrm{Nu}_{f}$, are shown. Figure 15 shows the total heat transfer from the hot wall to the system. This Nusselt number is defined as

$$
\begin{aligned}
\mathrm{Nu}_{\text {total }}= & \mathrm{Nu}_{m}+\mathrm{Nu}_{f}=-(1-\varepsilon) \lambda \int_{0}^{1}\left[\frac{\partial T_{m}^{*}}{\partial \xi}\right]_{\xi=0} d \eta \\
& -\varepsilon \int_{0}^{1}\left[\frac{\partial T_{f}^{*}}{\partial \xi}\right]_{\xi=0} d \eta
\end{aligned}
$$

The case of $\mathrm{Ra}=10^{6}, \mathrm{Da}=10^{-2}$, and $\mathrm{Ste}=1$ is considered first. Initially, at $\tau=0$, the heat transfer from the wall is large, but drops rapidly as both the metal and PCM heat up. In the presence of the foam, heat transfer is conduction dominated and at steady state, the dimensionless temperature gradient at the wall for both metal foam and PCM is unity. Hence, $\mathrm{Nu}_{\text {total }} \sim \varepsilon+(1-\varepsilon) \lambda$. This is true for both the $\mathrm{Nu}_{i, d}$ values plotted in Fig. 14. As the Rayleigh number is increased, the Nusselt number at the wall increases due to increased natural convection. The increase in the Nusselt number for metal foam is manifested as a distortion in the metal foam isotherms because of coupling with the PCM (see Figure 9(b)). The greatest contribution to sensible heat transfer from the hot wall is due to the metal. The fluid primarily contributes to the phase change heat transfer and heat from the hot wall is conveyed to it by the metal.

\section{Conclusions}

A parametric study of the transient melting of PCMs integrated into metal foams for enhanced effective thermal conductivity has been investigated for the case of a step change in boundary temperature. A number of important results have been obtained. For the range of parameters considered, for metal foams with interstitial Nusselt numbers $\mathrm{Nu}_{i, d}>5.9$, a single-temperature model is sufficient for analysis. But for smaller $\mathrm{Nu}_{i, d}$ values, the metal foam and the PCM are sufficiently out of equilibrium that a twotemperature model is necessary. The metal foam is seen to act in two ways. First, it substantially dampens convective flow because of frictional resistance. Second, since the metal responds far faster than the fluid, the linear temperature profile in the metal tends to imprint itself on the fluid, leading to conductionlike temperature profiles for $\mathrm{Ra} \leqslant 10^{6}$. Even at $\mathrm{Ra}=10^{8}$, only a mild departure from the conduction temperature profile is seen. As a result, the melt volume fraction at steady state, which is a measure of the total heat that can be absorbed, is approximately $\left(1-T_{\text {melt }}^{*}\right)$. This value may be less than that obtained without the metal foam because of the damping of convective flow. The metal foam acts to substantially decrease the response time of the system and may, thus, lead to far less overheating during the transient, despite the smaller melt volume fraction at steady state.

This study has investigated the transients due to a step change in boundary temperature. In many applications, periodic pulsed heating may be used. The difference in response time between systems with and without metal foam enhancers has important implications for the management of transient energy pulses. If the time scale of the energy pulse is short compared to the response time of the system, local overheating is possible. Since the metal foam response time is typically far faster than typical energy pulse time scales, it would tend to perform far better than systems without metal foams. These aspects must be investigated further to gain a clearer understanding of pulsed heating.

\section{Acknowledgment}

Support for this work from industry members of the Cooling Technologies Research Center, an NSF Industry/University Cooperative Research Center (www.ecn.purdue.edu/CTRC), is gratefully acknowledged.

\section{Nomenclature}

$$
\begin{aligned}
a_{s f} & =\text { specific surface area, } m^{-1} \\
\mathrm{C}_{\mathrm{p}} & =\text { isobaric specific heat, } \mathrm{JKg}^{-1} \mathrm{~K}^{-1} \\
\mathrm{Da} & =\text { Darcy number } \\
d & =\text { particle diameter or mean pore diameter, } \mathrm{m} \\
F & =\text { inertial coefficient } \\
\mathbf{g} & =\text { gravity vector, } \mathrm{ms}^{-2} \\
H & =\text { height of the enclosure, } \mathrm{m} \\
h_{v} & =\text { volumetric heat transfer coefficient, } \mathrm{Wm}^{-3} \mathrm{~K}^{-1} \\
K & =\text { permeability, } \mathrm{m}^{2} \\
k & =\text { thermal conductivity, } \mathrm{Wm}^{-1} \mathrm{~K}^{-1} \\
k_{e} & =\text { equivalent thermal conductivity, } \mathrm{Wm}^{-1} \mathrm{~K}^{-1} \\
\mathrm{Nu} & =\text { Nusselt number based on the porous foam } \\
& \text { height } \\
\mathrm{Nu}_{i} & =\text { interstitial Nusselt number }\left(h_{v} H^{2} / k_{f}\right) \\
\mathrm{Nu}_{i, d} & =\text { interstitial Nusselt number based on pore diam- } \\
\mathrm{P} & =\text { eter }\left(h_{v} d^{2} / k_{f}\right) \\
\mathrm{Pr} & =\text { Pressure, Nm }{ }^{-2} \\
\mathrm{Ra} & =\text { Rayleigh number } \\
\mathrm{Re} & =\text { Reynolds number } \\
\mathrm{Ste} & =\text { Stefan number } \\
T & =\text { temperature, } \mathrm{K} \\
t & =\text { time, } \mathrm{s} \\
\mathrm{U} & =\text { velocity vector, ms }{ }^{-1} \\
V & =\text { volume } \\
u, v & =\text { velocity in } x \text { and } y \text { directions, } \mathrm{ms}^{-1} \\
x, y & =\text { Cartesian coordinates } \\
&
\end{aligned}
$$

Greek Symbols

$$
\begin{aligned}
\alpha= & \text { thermal diffusivity, } \mathrm{m}^{2} \mathrm{~s}^{-1} \\
\beta= & \text { coefficient of thermal expansion, } \mathrm{K}^{-1} \\
\Delta H= & \text { enthalpy of freezing/melting, } \mathrm{JKg}^{-1} \\
\Delta T= & \text { mushy zone thickness, } \mathrm{K}^{-1} \\
\varepsilon= & \text { porosity } \\
\gamma= & \text { fraction of liquid melt in the PCM } \\
\eta= & \text { dimensionless } y \text { coordinate } \\
\varphi= & \text { fraction of liquid PCM }(=\varepsilon \gamma) \\
\lambda= & \text { ratio of thermal conductivities of metal foam } \\
& \text { and fluid } \\
\lambda_{e}= & \text { ratio of equivalent thermal conductivity to fluid } \\
\mu= & \text { thermal conductivity } \\
v= & \text { kinamic viscosity, } \mathrm{N} \mathrm{sm}^{-2} \\
\rho= & \text { density, } \mathrm{kg} \mathrm{m}^{-3}
\end{aligned}
$$


$\tau=$ dimensionless time

$\xi=$ dimensionless $x$ coordinate

$\Omega=$ ratio of volumetric heat capacities of metal foam and fluid

\section{Subscripts}

$$
\begin{aligned}
C & =\text { cold } \\
d & =\text { diameter } \\
e f & =\text { effective fluid property } \\
e q & =\text { equivalent } \\
f & =\text { fluid }(\mathrm{PCM}) \\
H & =\text { hot/height of enclosure } \\
l & =\text { liquid } \\
p & =\text { pore or particle } \\
m & =\text { metal foam } \\
s & =\text { solid (PCM) }
\end{aligned}
$$

\section{Superscripts}

$*$ = dimensionless quantity

$$
n=\text { time step } n
$$

\section{References}

[1] Sparrow, E. M., Patankar, S. V., and Ramadhyani, S., 1977, "Analysis of Melting in the Presence of Natural Convection in the Melt Region," ASME J. Heat Transfer, 99, pp. 520-526.

[2] Gau, C., and Viskanta, R., 1986, "Melting and Solidification of a Pure Metal on a Vertical Wall," ASME J. Heat Transfer, 108, pp. 174-181.

[3] Jany, P., and Bejan, A., 1988, "Scaling Theory of Melting With Natural Convection in an Enclosure," Int. J. Heat Mass Transfer, 31, pp. 1221-1235.

[4] Yao, L. S., and Prusa, J., 1989, "Melting and Freezing," Adv. Heat Transfer, 19, pp. 1-95.

[5] Beckermann, C., and Viskanta, R., 1988, "Natural Convection Solid/Liquid Phase Change in Porous Media," Int. J. Heat Mass Transfer, 31, pp. 35-46.

[6] Jany, P., and Bejan, A., 1988, "Scales of Melting in the Presence of Natural Convection in a Rectangular Cavity Filled With Porous Medium," ASME J. Heat Transfer, 110, pp. 526-529.

[7] Bejan, A., 1989, "Theory of Melting With Natural Convection in an Enclosed Porous Medium," ASME J. Heat Transfer, 111, pp. 407-415.

[8] Chellaiah, S., and Viskanta, R., 1990, "Natural Convection Melting of a Frozen Porous Medium," Int. J. Heat Mass Transfer, 33, pp. 887-899.

[9] Chellaiah, S., and Viskanta, R., 1990, "Melting of Ice-Aluminum Balls Systems," Exp. Therm. Fluid Sci., 3, pp. 222-231.

[10] Viskanta, R., 1991, "Phase Change Heat Transfer in Porous Media," Proc. of 3rd Int. Symp. on Cold Region Heat Transfer, University of Alaska, Fairbanks, pp. 1-24.

[11] Ellinger, E. A., and Beckermann, C., 1991, "On the Effect of Porous Layers on Melting Heat Transfer in an Enclosure," Exp. Therm. Fluid Sci., 4, pp. 619629

[12] Tong, X., Khan, J. A., and Amin, M. R., 1996, "Enhancement of Heat Transfer by Inserting a Metal Matrix into a Phase Change Material," Numer. Heat Transfer, Part A, 30, pp. 125-141

[13] Vesligaj, M. J., and Amon, C. H., 1999, “Transient Thermal Management of Temperature Fluctuations During Time Varying Workloads on Portable Electronics," IEEE Trans. Compon. Packag. Technol., 22, pp. 541-550.
[14] Alawadhi, E. M., and Amon, C. H., 2003, "PCM Thermal Control Unit for Portable Electronic Devices: Experimental and Numerical Studies," IEEE Trans. Compon. Packag. Technol., 26, pp. 116-125.

[15] Harris, K. T., Haji-Sheikh, A., and Agwu Nnanna, A. G., 2001, "Phase-Change Phenomena in Porous Media-A Non-Local Thermal Equilibrium Model," Indian J. Pure Appl. Phys., 44, pp. 1619-1625.

[16] Krishnan, S., Murthy, J. Y., and Garimella, S. V., 2004, "A Two-Temperature Model for Analysis of Passive Thermal Control Systems," ASME J. Heat Transfer, 126, pp. 628-637.

[17] Minkowycz, W. J., Haji-Sheikh, A., and Vafai, K., 1999, “On Departure From Local Thermal Equilibrium in Porous Media Due to a Rapidly Changing Heat Source: The Sparrow Number," Int. J. Heat Mass Transfer, 42, pp. 3373 3385.

[18] Nield, D. A., and Bejan, A., 1992, Convection in Porous Media, SpringerVerlag, New York.

[19] Vafai, K., and Sozen, M., 1990, "An Investigation of a Latent Heat Storage Porous Bed and Condensing Flow Through it," ASME J. Heat Transfer, 112, pp. 1014-1022.

[20] Kaviany, M., 1995, Principles of Heat Transfer in Porous Media, SpringerVerlag, New York.

[21] Boomsma, K., Poulikakos, D., and Zwick, F., 2003, "Metal Foams as Compact High Performance Heat Exchangers," Mech. Mater., 35, pp. 1161-1176.

[22] Price, D. C., 2003, "A Review of Selected Thermal Management Solutions for Military Electronic Systems," IEEE Trans. Compon. Packag. Technol., 26, pp. 26-39.

[23] Ashby, M. F., Evans, A., Fleck, N. A., Gibson, L. J., Hutchinson, J. W., and Wadley, H. J. G., 2000, Metal Foams: A Desgin Guide, ButterworthHeinemann, Boston.

[24] Amiri, A., and Vafai, K., 1994, "Analysis of Dispersion Effects and NonThermal Equilibrium, Non-Darcian, Variable Porosity Incompressible Flow Through Porous Media," Int. J. Heat Mass Transfer, 37, pp. 939-954.

[25] Hwang, J. J., Hwang, G. J., Yeh, R. H., and Chao, C. H., 2002, "Measument of Interstitial Convective Heat Transfer Coefficient and Frictional Drag for Flow Across Metal Foams," ASME J. Heat Transfer, 124, pp. 120-129.

[26] Ferziger, J. H., and Peric, M., 1995, Computational Methods for Fluid Dynamics, Springer-Verlag, Berlin.

[27] Wakao, N., and Kaguei, S., 1982, Heat and Mass Transfer in Packed Beds, Gordon and Beach, New York, NY.

[28] Calmidi, V. V., and Mahajan, R. L., 2000, "Forced Convection in High Porosity Metal Foams," ASME J. Heat Transfer, 122, pp. 557-565.

[29] Phanikumar, M. S., and Mahajan, R. L., 2002, "Non-Darcy Natural Convection in High Porosity Metal Foams," Int. J. Heat Mass Transfer, 45, pp. 37813793.

[30] Kuwahara, F., Shirota, M., and Nakayama, A., 2001, “A Numerical Study of Interfacial Convective Heat Transfer Coefficient in Two-Energy Equation Model for Convection in Porous Media,” Int. J. Heat Mass Transfer, 44, pp. 1153-1159.

[31] Morgan, V. T., 1975, "The Overall Convective Heat Transfer From Smooth Circular Cylinders," Adv. Heat Transfer, 11, pp. 199-264.

[32] Frankel, N. A., and Acrivos, A., 1968, "Heat and Mass Transfer From Small Spheres and Cylinders Freely Suspended in Shear Flow," Phys. Fluids, 11, pp. 1913-1918.

[33] Benard, C., Gobin, C., and Martinez, F., 1985, "Melting in Rectangular Enclosures: Experiments and Numerical Simulations," ASME J. Heat Transfer, 107, pp. 794-803.

[34] Krishnan, S., Murthy, J. Y, and Garimella, S. V., 2004 "A Two-Temperature Model for Solid/Liquid Phase Change in Metal Foams," Proc. ASME Heat Transfer/Fluids Engineering Summer Conf., ASME, New York, ASME Paper No. HT-FED2004-56337, Charlotte, NC, July 2004. 